\title{
MRNA-based Personalized Cancer Vaccine mRNA-4157
}

National Cancer Institute

\section{Source}

National Cancer Institute. mRNA-based Personalized Cancer Vaccine mRNA-4157. NCI

Thesaurus. Code C146813.

An mRNA-based individualized, therapeutic personalized cancer vaccine (PCV) targ eting twenty tumor-associated antigens (TAAs) that are specifically expressed by the patient's cancer cells, with potential immunostimulatory and antineoplastic activities. The cells from the patient's tumor are analyzed, and genetic sequencing is used to identify twenty neoantigen epitopes that may elicit the strongest immune response in the patient. The sequences encoding the twenty patient-specific epitopes are transcribed and loaded onto a single mRNA molecule. Upon administration, the mRNA-based PCV mRNA-4157 is taken up and translated by antigen presenting cells (APCs). Then, the expressed epitopes are presented via major histocompatibility complex (MHC) molecules on the surface of the APCs. This leads to an induction of both cytotoxic T-lymphocyte (CTL)- and memory T-cell-dependent immune responses that specifically target and destroy the patient's cancer cells that express these neoantigens. 